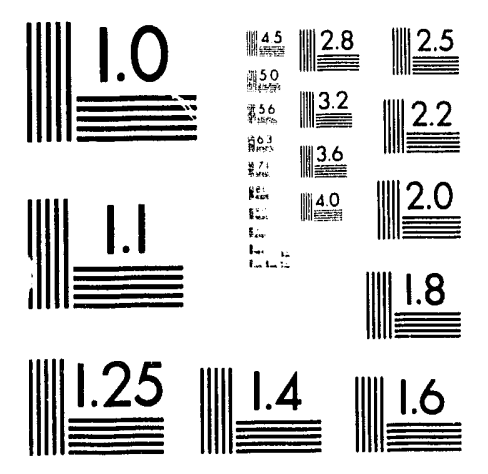



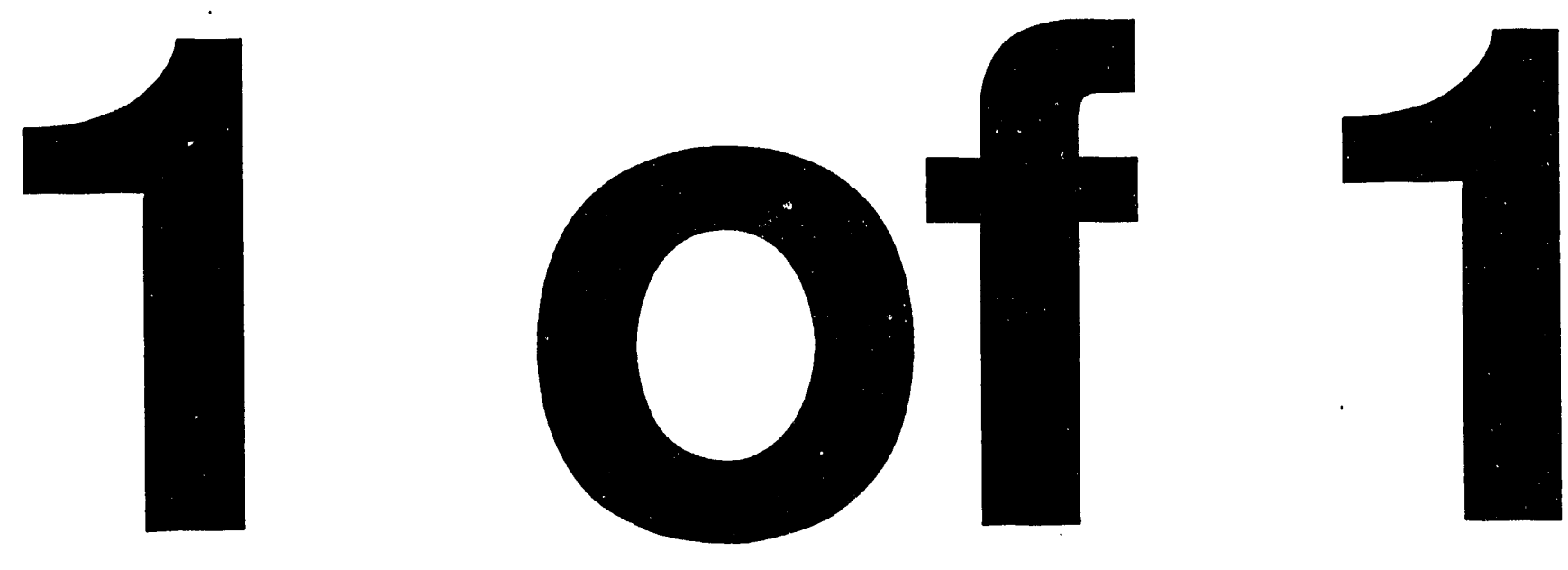


\title{
SYSTEM IDENTIFICATION USING A WAVELET-BASED APPROACH ${ }^{1}$
}

\author{
David A. Schoenwald
}

Paper to be presented at the 32nd IEEE Conference on Decision and Control

San Antonio, Texas

December 15-17, 1993

\section{DISCLAIMER}

\begin{abstract}
This report was prepared as an account of work sponsored by an agency of the United States Government. Neither the United States Government nur any agency thereof, nor any of their employees, makes any warranty, express or implied, or assumes any legal liability or responsibility for the accuracy, completeness, or usefulness of any information, apparatus, product, or process disclosed, or represents that its use would not infringe privately owned rights. Reference herein to any specific commercial product, process, or service by trade name, trademark, manufacturer, or otherwise does not necessarily constitute or imply its endorsement, recommentation, or favoring by the United States Government or any agency thereof. The views and opinions of authors expressed herein do not necessarily state or reflect those of the United States Government or any agency thereof.
\end{abstract}

\footnotetext{
${ }^{1}$ Research sponsored by the Laboratcry Directed Research Program of Oak Ridge National Laboratory, managed for the Department of Energy by Martin Marietta Energy Systems, Inc., under Contract No. DE-AC05-84OR21400.
} 


\title{
SYSTEM IDENTIFICATION USING A WAVELET-BASED APPROACH ${ }^{\dagger}$
}

\author{
David A. Schoenwald \\ Instrumentation and Controls Division \\ Oak Ridge National Laboratory \\ P. O. Box 2009 \\ Oak Ridge, TN 37831-8066
}

\begin{abstract}
In this paper, an approach to system identification using the wavelet transform is outlined. The method is based on system identification algorithms that belong to the class of modulating function methods. In particular, the Pearson-Lee method is modified to make use of wavelet basis functions which provide a more precise time window to process the plant input-output data. It is proposed here that replacing these modulating functions with wavelet basis functions will provide data filtering which is less sensitive to noise and initial conditions.
\end{abstract}

\section{Background}

The field of wavelet analysis has garnered a great deal of attention in the engineering literature during the last few years due to its power and utility in analyzing signals (see [4] for a survey). The subject has found applications in signal and image processing, machine vision, and data compression. One of the most powerful analytical tools associated with wavelet analysis is that of the Wavelet Transform (WT). The WT is a variation of the windowed Fourier Transform (FT) in which the window size is varied with frequency. The WT uses short (in time) windows at high frequencies and long windows at low frequencies. This is intuitive, because at high frequencies, one would like to resolve the fast time-varying spectra with a finer time window. Likewise, at lower frequencies, one would prefer a longer time window since the signal components are not changing as rapidly. The windowed FT, regardless of the shape of the window chosen, does not provide this feature since there is no mechanism for varying window sizes.

The WT is especially useful in analysis of nonstationary signals. Abrupt changes in statistical properties of signals are spread out over a large frequency range in the transformed signal. Thus, a 'local' FT is essential in viewing the signal at a time range at which it is approximately stationary. The WT accomplishes this (as does a short time FT), but it also provides the variable resolution needed for a spectrally diverse signal. This paper focuses on techniques that can apply the advantages of the WT to the problem of system identification.

\footnotetext{
†Research sponsored by the Laboratory Directed Research Program of Oak Ridge National Laboratory, managed for the Department of Energy by Martin Marietta Energy Systems, Inc., under Contract No. DE-AC05-84OR21400.
}

The problem of system identification using the WT has been addressed by several researchers. Pati and Krishnaprasad [2] have analyzed the problem of rational wavelet approximations to stable linear systems using the theory of frames. They have developed a system identification algorithm based on truncation of these wavelet series representations for a linear system. Tsatsanis and Giannakis [5] have looked at the problem of system identification for time-varying systems using wavelets. Work by Willsky et al. [6] has analyzed the problem of identifying stochastic processes using multisenle representations and dyadic trees. In this paper, we are concerned with the identification of time-invariant linear systems and polynomial nonlinear systems. The Pearson-Lee method serves as a starting point for our approach and is outlined in the next section.

\section{System Jdentification}

The class of modulating function methods are distinguished by two characteristics [1]: (1) Ability to handle arbitrary (not fixed) initial conditions and (2) Avoiding differentiation of experimental data. This last feature is important when noise is encountered. The modulating functions instead of the plant signals bear the burden of differentiation. The Pearson-Lee method [3] specifically uses trigonometric functions as modulating functions so as to allow the use of Fast Fourier Transforms (FFTs) in evaluating numerical integrations. The details of how the method works can be found in $[1,3]$, but the following outline summarizes the procedure.

(1) Given a finite time series of input and output data over a time window of length $T$, multiply both sides of the assumed system model equation (with unknown coefficients) by the $i$ th modulating function (there will be at least as many modulating functions as unknown parameters).

(2) Integrate both sides of the system model equation over the time window of length $T$.

(3) Utilizing integration by parts, the modulating functions and input-output series are interchanged, that is, the modulating functions will be operated upon by derivatives with the input-output data appearing outside the differentiation operation.

(4) The integrals are replaced by symbols resulting in a matrix linear equation that can be solved for the unknown parameters using singular value decompositions or least squares fitting techniques.

(5) The integrals are evaluated using FFTs since the modulating functions will be sinusoidal and thus 
smooth.

(6) A candidate set of modulating functions is chosen such that they (along with their first derivatives) are periodic over the data window (these functions will not be unique).

The contribution of this paper is proposing that the modulating function be replaced with a wavelet basis function. The modulating function acts as a filter over the data window. However, as with the $F^{\prime} \Gamma$ itself, this function is infinite in extent and does not work well with nonstationary plant signals. A wavelet modulating function will provide a more precise data filter with variable resolution. The wavelet basis function can still be chosen to satisfy the periodicity conditions, but it must have sufficiently fast decay outside the data window to have compact support.

Consider a wavelet basis function $\phi(t)$ chosen to be a modulating function. For simplicity, consider a simple second-order linear time-invariant system

$\ddot{y}+a_{1} \dot{y}+a_{2} y=b_{1} \dot{u}+b_{2} u$

in which the objective is to estimate the four parameters $a_{1}, a_{2}, b_{1}$, and $b_{2}$ using input-output data $u(t)$ and $y(t)$ over a finite time window ranging from $t=0$ to $t=T$. Now multiply both sides of (1) by $\phi_{i}(t)$ allowing for time shifting and scaling then integrating over the data window. With this, one obtains (via integration by parts)

$$
\begin{aligned}
& \int_{0}^{T} y \ddot{\phi}_{i}((t-\tau) / a) d t+a_{1} \int_{0}^{T} y \dot{\phi}_{i}((t-\tau) / a) d t \\
+ & a_{2} \int_{0}^{T} y \phi_{i}((t-\tau) / a) d t \\
= & b_{1} \int_{0}^{T} u \dot{\phi}_{i}((t-\tau) / a) d t \\
+ & b_{2} \int_{0}^{T} u \phi_{i}((t-\tau) / a) d t
\end{aligned}
$$

where $a$ is the scaling parameter, $\tau$ is the time-shift parameter, and $i=1, \ldots, 4$. Define the following integrals

$$
\begin{aligned}
& p_{i}(\tau, a)=\int_{0}^{T} y \ddot{\phi}_{i}((t-\tau) / a) d t \\
& q_{i}(\tau, a)=\int_{0}^{T} y \dot{\phi}_{i}((t-\tau) / a) d t \\
& r_{i}(\tau, a)=\int_{0}^{T} y \phi_{i}((t-\tau) / a) d t \\
& g_{i}(\tau, a)=\int_{0}^{T} u \dot{\phi}_{i}((t-\tau) / a) d t \\
& h_{i}(\tau, a)=\int_{0}^{T} u \phi_{i}((t-\tau) / a) d t
\end{aligned}
$$

which are then combined with (2) to yield the following linear matrix equation $\cdot\left[\begin{array}{llll}q_{1} & r_{1} & g_{1} & h_{1} \\ q_{2} & r_{2} & g_{2} & h_{2} \\ q_{3} & r_{3} & g_{3} & h_{3} \\ q_{4} & r_{4} & g_{4} & h_{4}\end{array}\right]\left[\begin{array}{c}-a_{1} \\ -a_{2} \\ b_{1} \\ b_{2}\end{array}\right]=\left[\begin{array}{l}p_{1} \\ p_{2} \\ p_{3} \\ p_{4}\end{array}\right]$

that is to be solved for the unknown plant coefficients. Note that the resultant plant parameter estimates will depend on the wavelet scale factor, $a$, and the shift parameter, $\tau$.

Hence, the parameter estimates are solved for via a minimum variance or expected value criterion. That is, the variables $a$ and $\tau$ serve as optimizing parameters to determine plant coefficients that best characterize the plant despite the presence of disturbances or time delays. This optimizing procedure can be done in one of several ways. First, a performance criterion minimizing variance based on known plant noise statistics is established. Then (8) is solved via least squares or singular value decompositions. Second, the plant parameter estimates can be chosen to vary with time for a process that undergoes widely varying operating conditions. Each operating condition would have its own admissible parameter set reflecting the time-varying nature of processes such as chemical plants. Finally, a set of plant parameters can be chosen by applying an expected value operator to the estimates from (8) where the expected value is with respect to $a$ and $\tau$. Work in progress is analyzing these different approaches with respect to noise and changes in operating conditions as well as efficient numerical methods for putting these techniques into practice.

\section{References}

[1] T. B. Co and B. E. Ydstie, "System identification using modulating functions and Fast Fourier Transforms," Computers and Chemical Engineering, vol. 14, no. 10, pp. 1051-1066, 1990.

[2] Y. C. Pati and P. S. Krishnaprasad, "Analysis and synthesis of feedforward neural networks using discrete affine wavelet transforms," IEEE Transactions on Neural Networks, 1992 (Also Univ. of Md., Systems Research Center Technical Report SRC-TR-90-44).

[3] A. E. Pearson and F. C. Lee, "On the identification of polynomial input-output differential systems," IEEE Transactions on Automatic Control, vol. AC-30, no. 8, pp. 778-782, 1985.

[4] O. Rioul and M. Vetterli, "Wavelets and signal processing," IEEE SP Magazine, pp. 14-38, October 1991 .

[5] M. K. Tsatsanis and G. B. Giannakis, "Timevarying system identification using wavelets," Proceedings of the 26th Asilomar Conference on Signals, Systems, and Computers, pp. 125-129, Pacific Grove, CA, October 1992.

[6] A. S. Willsky, K. C. Chou, M. Basseville, and A. Benveniste, "Wavelet transforms, mu!'ircsolution dynamical models, and multigrid estimation algorithms," Proceedings of the 11th IFAC World Congress, Tallinn, Estonia, pp. 143-147, August 1990 . 

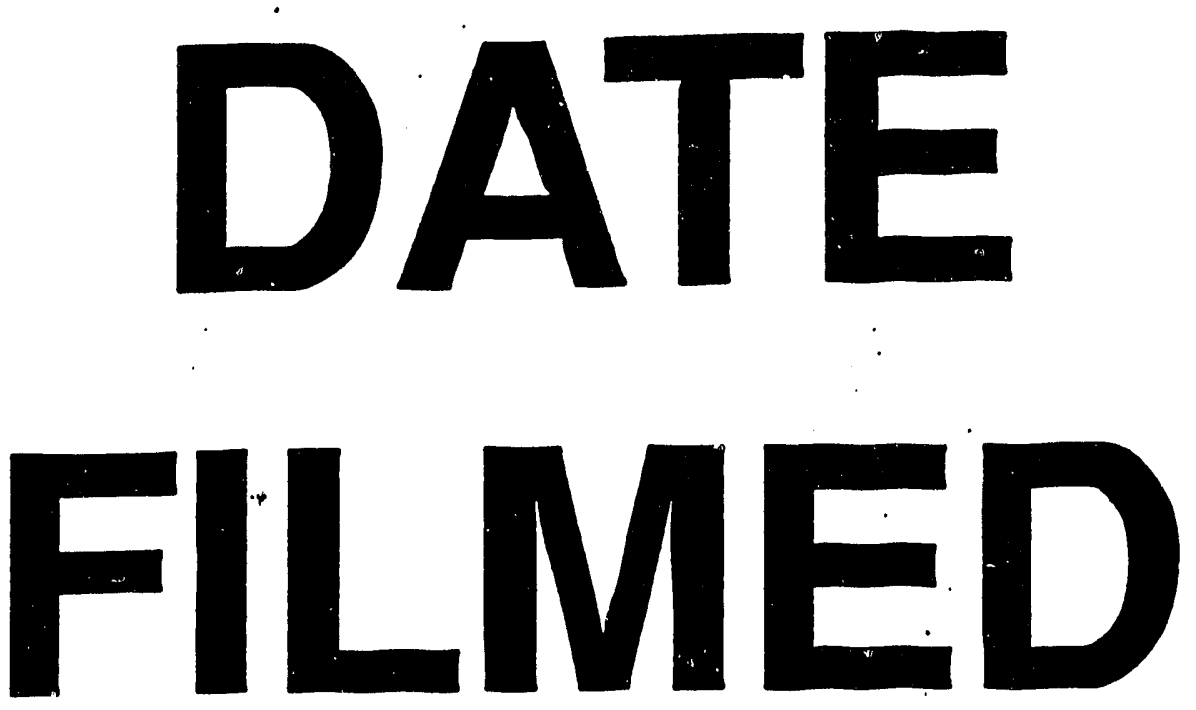

$12 / 14 / 93$
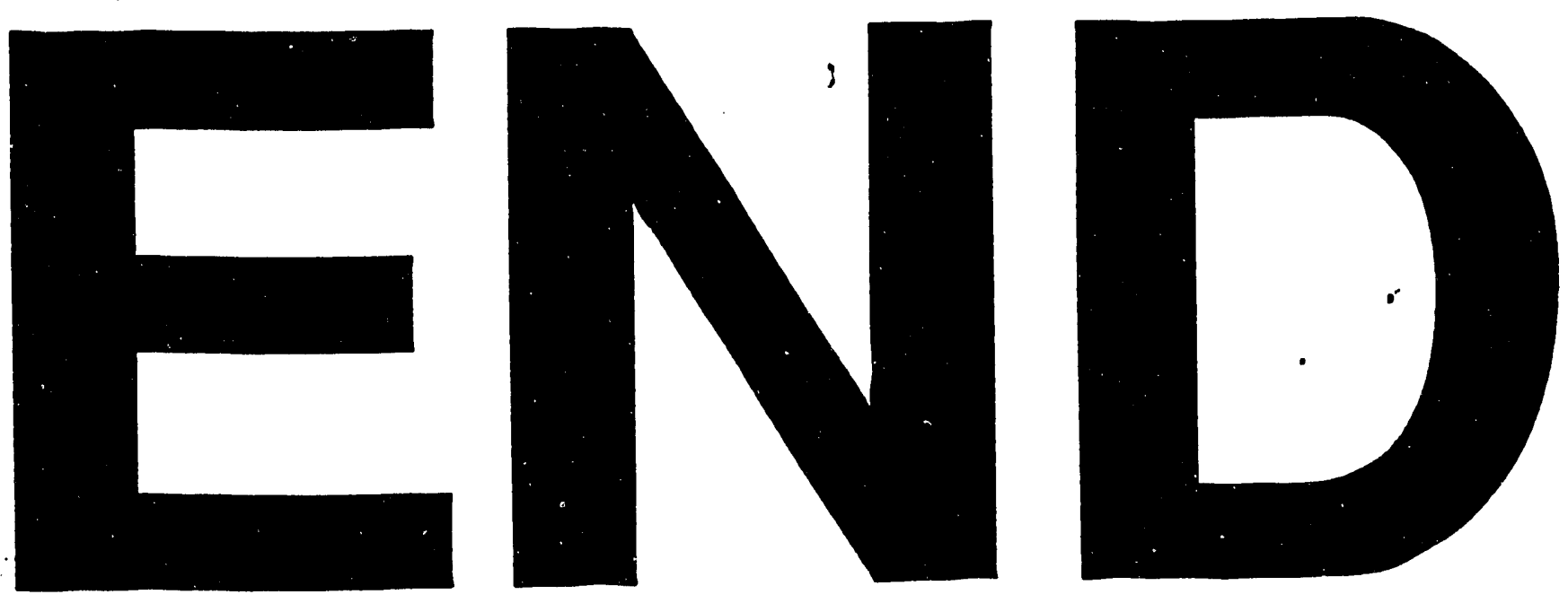
